\title{
Embryobildung bei Balanophora.
}

\author{
Von A. Ernst.
}

(Mit Tafel I und II.)

Im Jahre 1900 hat Juel am Beispiel von Antennaria alpina den genauen Verlauf einer Embryobildung ohne vorhergegangene Befruchtung beschrieben. Seither sind ähnliche Fortpflanzungsvorgänge bei Vertretern der verschiedensten Verwandtschaftskreise aufgefunden worden. Winkler hat (1908, pag. 294) sowohl die sicheren Fälle von Parthenogenesis und Apogamie, als auch die unsicheren und nachzuprüfenden Angaben über zahlreiche andere Fälle zusammengestellt und besprochen und jüngst in einer neuen Zusammenstellung (1913, pag. 265) auch die in den letzten Jahren bekannt gewordenen Beispiele herangezogen.

In der Mehrzahl der bis jetzt eingehend untersuchten Fälle von Embryobildung ohne Befruchtung bei Angiospermen handelt es sich um somatische Parthenogenesis, um Entstehung eines Embryos aus einer unbefruchteten Eizelle mit nicht reduzierter Chromosomenzahl. Viel seltener ist bei den Angiospermen somatische Apogamie festgestellt worden. Bei einigen Alchemilla-Arten, Allium odorum, Burmannia coelestis, erfolgt gelegentlich neben Embryobildung aus der Eizelle auch Embryobildung aus einer Synergide oder Antipode. In solchen Fällen kommt es also zu einer gelegentlichen Polyembryonie, während habituelle Polyembryonie in der Regel auf Erzeugung von Adventivembryonen aus nicht dem Embryosack angehörigen Elementen zurückzuführen ist.

Auch die Endospermbildung erfolgt in den Fällen parthenogenetischer und apogamer Embryobildung ohne Mitwirkung eines männlichen Kernes. In den meisten Fällen geht dabei der Bildung des Endosperms eine Verschmelzung der beiden Polkerne zum sekundären Embryosackkern voraus. Bei Antennaria alpina (Juel 1900, pag. 24) treten aber die beiden Polkerne ohne vorausgegangene Verschmelzung ungefähr gleichzeitig in Teilung. Bei Helosis und Balanophora geht die Endospermbildung ausschließlich vom einen Polkern aus und für zwei Arten der letzteren Gattung, Balanophora Flora, Bd. 106. 
elongata Blume und B. globosa Junghuhn findet sich in der Literatur die Angabe, daß dieser eine Kern nicht nur dem Endosperm, sondern auch dem Embryo den Ursprung gebe.

Für B. elongata Bl. sind diese Verhältnisse von M. Treub 1898 in einer ausführlichen und mit zahlreichen Tafeln belegten Abhandlung beschrieben worden.

Wie bei anderen Balanophoraceen sind auch bei B. elongata die zu auffälligen Blütenständen vereinigten männlichen und weiblichen Blüten in Bau und Entwicklung außerordentlich reduziert. Im besonderen gilt dies für die weiblichen Blüten, in denen es weder zur Bildung einer Blütenhülle, noch zur Differenzierung eigentlicher Fruchtblätter kommt. Die jugendliche Blüte besteht ausschließlich aus einem papillenartigen Zellkörper, an dessen Scheitel später durch intensive Teilungstätigkeit einzelner Oberflächenzellen ein langer, griffelähnlicher Fortsatz auswächst. Da beim weiteren Wachstum die Basis sich in einen stielförmigen Träger und einen bauchartig angeschwollenen Teil differenziert, so erinnert das weibliche Organ von Balan ophora auffallend an ein Moosarchegonium, von dem es sich nur durch das Fehlen eines Halskanals wesentlich unterscheidet. Im bauchigen Teil dieses Organs entsteht subepidermal der Embryosack. Seine Entwicklung geht bis zum achtkernigen Stadium normal vor sich. An den beiden Polen des U-förmig gekrümmten Sackes sind die Kerne in den bekannten Tetraden angeordnet. Die am Antipodenende gelegenen Kerne gehen, ohne daß es zur Bildung von Antipodenzellen kommt, bald zugrunde. Am anderen Ende entsteht ein Eiapparat, dessen Zellen nach Treub's Darstellung ebenfalls bald abortieren, während der dazugehörige Polkern, der sich schon vorher durch besondere Größe von den übrigen Kernen auszeichnete, erhalten bleibt. Verschmelzung mit dem Polkern der anderen Tetrade findet nicht statt. $\mathrm{Er}$ tritt in Teilung und liefert einen aus großen Zellen bestehenden Gewebekörper, das Endosperm. Eine zentrale Zelle dieses Endosperms wird nach Treub zur Mutterzelle des Embryos, der also nach dieser Darstellung apogam aus dem als Prothallium zu deutenden Endosperm hervorgeht.

Speziell in der Darlegung der Befruchtungsverhältnisse und der Embryobildung stehen die Befunde Treub's in starkem Widerspruch zu allen älteren Untersuchungen an Balanophoraceen, im besonderen zu denjenigen Hofmeister's (1859, pag. 572), sowie einer kurz vorher erschienenen Arbeit von Van Tieghem über B. indica (1896, pag. 295). Trotzdem ist meines Wissens in der neueren Literatur 
niemals ein Zweifel gegenüber den Angaben Treubs geäußert worden, auch nicht von $\mathrm{V}$ an Tiegh em, obschon dieser bei einer erneuten Untersuchung von B. indica (1907, pag. 174) seine früheren Angaben durchaus bestätigt fand. Dies ist auch begreiflich. Die Treub'sche Beweisführung, speziell diejenige über das Schicksal des Eiapparates, macht den Eindruck vollkommener Lückenlosigkeit, und zur anerkarnten Autorität Treub's in embryologischen Dingen kam hinzu, daß nur ein Jahr später Lotsy für B. globosa Jungh. völlig übereinstimmende Resultate veröffentlicht hat. So ist es $\mathrm{zu}$ verstehen, daß das ungewöhnliche Verhalten von Balanophora - apogame Embryobildung aus einer Endospermzelle - in den Darstellungen der Embryologie der Angiospermen vielfach beschrieben und zitiert ${ }^{1}$ ) worden ist und auch in dem berühmten Streit um die phylogenetische Deutung des Endosperms eine große Rolle spielt.

Bei der Untersuchung der Embryosackentwicklung und der in einigen Fällen parthenogenetischen oder apogamen - Embryobildung javanischer Saprophyten hatte ich mich in den letzten Jahren vielfach mit Präparaten zu beschäftigen, die an Figuren aus dem von Treub und Lotsy dargestellten Entwicklungsgang des Embryos von Balanophora erinnerten. Sie weckten die Hoffnung, auf neue Beispiele für apogame Embryobildung aus Endosperm hinweisen zu können. So fand z. B. H. Wirz (1910, pag. 395), der unter meiner Leitung die Untersuchung der Embryosack- und Embryoentwicklung einer javanischen Sciaphila-Art ausführte, in älteren Samenanlagen stets wenigzellige Embryonen inmitten des Endospermgewebes ${ }^{2}$ ). Ähnliche Bilder erhielt ich selbst bei einer gleichzeitig vorgenommenen, aber nicht völlig abgeschlossenen und noch nicht publizierten Untersuchung über Cotylanthera

1) Siehe z. B. Coulter, J. M. and Chamberlain, Ch. J., Morphology of Angiosperms, 1903, pag. 218. Engler, A. und Gilg, E., Syllabus der Pflanzenfamilien, 1912, VII. Aufl., pag. 175. Goebel, K., Organographie der Pflanzen, 1898-1901, pag. 805. Knu th, P., Handbuch der Blütenbiologie, III, 1904, pag. 262. Strasburger, Ed., Einige Bemerkungen zur Frage nach der ,doppelten Befruchtung" bei den Angiospermen, 1900, pag. 315. Tischler, G., Über die Entwicklung der Samenanlagen in parthenokarpen Angiospermenfrüchten, 1913, pag. 7. v. Wettstein, R, Handbuch der systematischen Botanik, 1911, II. Aufl., pag. 456. Winkler, H., Über Parthenogenesis und Apogamie im Pflanzenreich, 1908, pag. 356 ; 1913, pag. 267.

2) Ähnliche Angaben sind übrigens in der Literatur nicht allzu selten. Um bei den tropischen Parasiten zu bleiben, sei z. B. an eine in der klassischen Arbeit Browns über Rafflesia enthaltene Notiz über die Samen von Hydnora erinnert, in der es heißt (1834). 
ten uis. Dann gelang aber, zunächst bei der letzteren Pflanze, nach eifrigem Bemühen auch bei Sciaphila der Nachweis, daß in jüngeren Entwicklungsstadien die Embryonen mit einem schmalen Embryoträger bis an die Oberfläche des Endosperms reichen, in älteren Stadien dagegen die Trägerzellen infolge Resorption entweder ganz verschwunden oder doch sehr undeutlich geworden sind. Auch in den jüngeren Samenanlagen waren überzeugende Bilder recht selten $\mathrm{zu}$ finden, da infolge der verschiedenen Orientierung der Samenanlagen im Fruchtknoten bei der Anfertigung von Schnitten nur wenige derart getroffen werden, daß auch der dünne, fast fadenartige Embryoträger median durchschnitten wird.

Immerhin ließ sich für beide Gattungen, Sciaphila und Cotylanthera. der einwandfreie Nachweis erbringen, daß der Embryo aus der Eizelle hervorgeht, die Befruchtung bei Cotylanthera sicher, bei Sciaphila sehr wahrscheinlich ausbleibt, und also Fälle somatischer Parthenogenesis vorliegen. Weiter ergab die Untersuchung dieser Gattungen, wie auch verschiedener Burmannia-Arten, daß die Weiterentwicklung der Eizelle im Vergleich zur Endospermentwicklung sehr spät einsetzt. Es geht ihr sogar meistens eine starke Volumenabnahme der Eizelle voraus. Später aber setzt das Wachstum der Keimzelle wieder mit Plasmavermehrung und Vergrößerung des Kernes ein; die nachfolgende Teilungstätigkeit allerdings bleibt minim und kommt schon nach Erzeugung eines wenigzelligen Embryokörpers zum Stillstand. Alle diese Befunde drängten zur Vermutung, daß vielleicht auch bei Balanophora ähnliche Verhältnisse vorliegen und trotz sorgfältiger Untersuchung Treub entgangen sein könnten. Eine Nachuntersuchung erschien also gerechtfertigt.

Sie wurde zunächst an Material beider Arten begonnen, das ich selbst 1905/06 im Gedehgebirge auf Java (s. a. Koorders 1912, Bd. II, pag. 177), am Wege von der Unterkunftshütte von Kanvon Kandang-Badak zum Gedehkrater gesammelt und, allerdings nur zu Demonstrationszwecken und kursorischen Übungen, in ca. $96^{\circ} \%$ igem Alkohol fixiert hatte. Es lieferte weder für B. el ong a t a noch für B. gl o b o sa alle Stadien, die zur Entscheidung der Frage notwendig waren. Weiteres Material vom selben Standorte, sowie aus der Umgebung von Tjibodas

„Enclosed in the albumen a perfectly spherical embryo is found, consisting entirely of a more minute and much less dense cellular tissue. On the surface of this embryo I have observed no point marking original attachment, nor any indication of a channel connecting it with the surface of the albumen, in the centre of which it is seated" (l. c. 228 u. Taf. XXX, Fig. 5 u. 7). 
wurde im November und Dezember 1910 von Prof. Dr. G. Senn, im Mai 1911 von Dr. Ch. Bernard für mich gesammelt und teils in Alkohol, teils im Gemisch von Carnoy fixiert ${ }^{1}$ ). Es sei mir gestattet, den beiden befreundeten Kollegen auch an dieser Stelle meinen besten Dank für ihre Bemühungen auszusprechen.

Erst nach der zeitraubenden Durcharbeitung des so zusammengekommenen reichhaltigen Materiales hat sich die Reihe meiner Belege dermaßen geschlossen, daß ich in der Lage bin, für die Richtigkeit der längst gehegten Vermutung den Beweis antreten zu können. Der hohen Autorität Treub's glaube ich eine eingehende Darlegung und Diskussion aller in Betracht kommenden Verhältnisse schuldig zu sein. Ich schicke daher der Darstellung der abweichenden Befunde in der Endosperm- und Embryoentwicklung eine kurze Darstellung der Embroysackentwicklung voraus, welche dartun soll, daß in allen anderen Punkten meine Nachuntersuchung die Ergebnisse Treub's vollständig bestätigt hat.

\section{Entwicklung des Embryosackes von B. elongata und B. globosa bis zum achtkernigen Stadium.}

Auf Entwicklung und Bau der Blüte und Blütenstände von Balanophora soll hier nicht eingetreten werden. Sie sind in den zitierten Arbeiten von Van Tieghem, Treub und Lotsy eingehend dargestellt und diskutiert worden. Auch die von Treub für B. elongata beschriebene Entwicklung des achtkernigen Embryosackes ist nicht nur von Lotsy durch die Untersuchung von B. globosa bestätigt worden, sondern stimmt in den Hauptzügen auch mit den Angaben von van Tieghem über B. indica überein. Ich kann mich daher im Nachfolgenden mit einer kürzeren Darstellung dieser ersten Entwicklungsvorgänge begnügen, in die nur ausführlichere Angaben über einige bis jetzt wenig berücksichtigte Stadien eingeflochten werden sollen. Zur Illustration derselben sei auf die Figuren von Taf. I, im übrigen auf das reiche Bildermaterial Treub's verwiesen.

1) Über die Zugehörigkeit eines Teiles der von Prof. Senn und Dr. Bernard gesammelten Materialien zu B. elongata oder B. globosa vermag ich keinen vollkommen sicheren Aufschluß zu geben, da nur die nackten Blütenstände fixiert worden waren und diese, wenigstens in jüngeren Stadien, bei beiden Arten sehr ähnlich sind. In beiden Materialsendungen herrschen weibliche Stände vor, die der Form nach B. elongata angehören. Im weiteren Verlauf der Untersuchung habe ich mich hauptsächlich an diese gehalten und von den mehr kugeligen, vermutlich der B. globos a angehörenden Ständen nur einige wenige zum Vergleich herangezogen, die dann zufälligerweise gerade sehr wichtige Stadien ergaben. 
Schon an sehr jungen Blütenständen sind in den kleinen Höckern, die an der Oberfläche der Achse entstehen, die Embryosackmutterzellen in subepidermaler Lagerung deutlich zu erkennen. Sie sind zunächst nicht viel größer als die umgebenden Zellen und unterscheiden sich von ihnen vorläufig nur durch dichteres und stärker färbbares Plasma. Später wird dieses vakuolig (Taf. I, Fig. 1) und der Kern nimmt an Größe zu. Er erreicht 10-16 $\mu$ Durchmesser.

In allen bisherigen Untersuchungen über Balanophoraceen fehlt, wie Winkler (1908, pag. 359) bemerkt, die Angabe, ob im Verlauf der Makrosporenentwicklung eine Reduktionsteilung durchgeführt werde oder nicht. Obschon sich die bisherigen Untersuchungen hierüber gar nicht äußern, erscheint es Winkler auf Grund der gegebenen Abbildungen doch sehr wahrscheinlich, daß die Reduktionsteilung unterbleibt, die Entwicklung des Embryosackinhaltes also mit somatischer Chromosomenzahl der Kerne stattfindet. In meinen Präparaten sind nun eine größere Anzahl von Mutterzellen mit Vorbereitungsstadien zur Kernteilung und andere mit Kernspindeln vorhanden. Mit ziemlicher Sicherheit vermag ich anzugeben, daß ein typisches Synapsisstadium, wie bei den meisten anderen parthenogenetischen Angiospermen, nicht zur Ausbildung gelangt. Auch den vorgeschritteneren Teilungsstadien gehen die bekannten Merkmale der heterotypischen Teilung völlig ab. Leider sind sämtliche Mutterzellen mit Spindelfiguren in meinen Präparaten so orientiert, daß die Teilungsfiguren von der Seite sichtbar sind. Es ist mir daher ebensowenig wie Treub gelungen, die Zahl der kleinen, fast kugeligen Chromosomen zu zählen, die zu einem gleichmäßig breiten Ring vereinigt sind. Unglücklicherweise war es auch trotz Untersuchung zahlreicher männlicher Blütenstände nicht möglich, die Teilung der Pollenmutterzellen ausfindig zu machen und eine kleinere Anzahl von Kernteilungsbildern in Pollenkörnern waren wiederum nicht deutlich genug, um eine einwandfreie Zählung der Chromosomen zu ermöglichen.

Die Teilung des Kernes der Embryosackmutterzelle findet bald in der Nähe der unteren (Taf. I, Fig. 2), bald der oberen Schmalseite der sich in die Länge streckenden Zelle statt. Es folgt derselben, vielleicht in etwas mehr als der Hälfte der Samenanlagen, eine Teilung der Mutterzelle in zwei Tochterzellen nach. In den anderen Samenanlagen unterbleibt die Teilung und die Mutterzelle entwickelt sich direkt als Embryosack weiter. Erfolgt eine Teilung, so sind die beiden Tochterzellen nur ausnahmsweise gleich groß (Taf. I, Fig. 5). Die zur Weiterentwicklung bestimmte wird in der Regel von vorneherein be- 
deutend größer angelegt. Besonders häufig ist die in Fig. 6 und 7 dargestellte Art der Zellteilung, die einer (Taf. I, Fig. 2) am Basalende der Mutterzelle erfolgten Kernteilung nachfolgt. Einen entsprechenden Teilungsverlauf der Embryosackmutterzelle in zwei Tochterzellen hat auch Lotsy für B. globosa angegeben. Er führt im Anschluß daran weiter aus, daß in solchen Fällen häufig beide Tochterzellen zu Embryosäcken auswachsen, die ihre Entwicklung gleichmäßig mit der Bildung von Endosperm und Embryo abschließen. Das ist in dem von mir untersuchten Material sicher nicht der Fall. Nur in einer einzigen Samenanlage wurden zwei ungefähr gleich stark ent wickelte, vierkernige Embryosäcke beobachtet.

Mit dem weiteren Wachstum des zweikernigen Embryosackes ist die eigenartige Gestaltsveränderung verknüpft, die Treub und van Tieghem unabhängig voneinander festgestellt haben. Sie ist von Treub in ihrem ganzen Verlauf sorgfältig beobachtet und in einer größeren Anzahl von Figuren genau dargestellt worden. Ich kann mich daher begnügen, in den Figuren 4 und 8 (Taf. I) Anfangs- und Endstadium dieses Wachstumsprozesses nochmals wiederzugeben. Der Embryosack wird zu einem annähernd U-förmigen Gebilde, dessen dicht nebeneinander liegenden Äste nach vorn gekrümmt und meistens ungleich lang sind. Schon zu Beginn dieses Wachstums erfolgt die Vereinigung aller Vakuolen des Embryosackes zu einem einheitlichen Saftraum und damit die Zurückdrängung des Plasmas an die Wand; die Hauptmasse desselben erfüllt kappenartig die Enden der scheitelwärts gerichteten Embryosackäste.

Es würde zu weit vom Thema abführen, hier der Ursache und der Bedeutung dieser eigentümlichen Wachstumserscheinung nachzuspüren. Treub, Goebel und Van Tieghem haben darüber Verschiedenes berichtet. Der letztere sieht in der Krümmung des Embryosackes einen erfolgreichen Versuch, das ursprünglich basalwärts gerichtete Eiende des Embryosackes dem befruchtenden Pollenschlauche entgegenzuführen. Wie man sich im übrigen zu dieser Deutung stellen will, so wird zuzugeben sein, daß die Aufwärtskrümmung des zuerst basalwärts gerichteten Poles jedenfalls in Beziehung steht $\mathrm{zu}$ der starken Reduktion, die in der Gesamtausbildung der weiblichen Blüte von Balanophora eingetreten ist. Es ist daher nicht zulässig, den Embryosack von Balanophora (Porsch 1907, pag. 37) in einer phylogenetischen Reihe, welche die allmähliche Reduktion einer Vielzahl von Archegonien beim Übergang von den Gymnospermen zu den Angio- 
spermen zeigen soll, als Beispiel eines primitiveren Embryosackes mit zwei Archegonien an demselben Pole aufzufassen.

An beiden Polen folgen nun die noch fälligen Kernteilungen rasch aufeinander, so daß jederseits vier Kerne gebildet werden. Die Zellbildung folgt an dem den Eiapparat bildenden Ende bald nach, während sie am Antipodenende meistens ausbleibt (Taf. I, Fig. 10-12). Auch Treub und Lotsy (pag. 181) haben am antipodialen Ende des Embryosackes nur nackte Kerne gefunden. Sie geben ferner an, daß an diesem Ende die letzte Teilung ziemlich häufig unterbleibt und anstatt vier Kernen nur deren zwei vorhanden sind. Das Antipodialende ist meistens plasmaärmer als das Eiende. Das Plasma erfüllt nicht immer die Spitze des Antipodenastes, sondern bildet zuweilen unter einer endständigen Vakuole einen gleichmäßig breiten Gürtel (Taf. I, Fig. 9 und 11). Die in diesem enthaltenen Kerne sind zunächst alle gleichgroß und auch in der Struktur nicht voneinander verschieden.

Drei derselben entsprechen den Antipodenkernen. Sie bleiben klein. Der vierte nimmt später etwas an Größe zu und bleibt, wie ich im Gegensatz zu Treub und Lotsy in einer größeren Anzahl von Embryosäcken feststellen konnte, auch nach der Degeneration und dem völligen Verschwinden der Antipodenkerne noch längere Zeit erhalten. Er wird also als Polkern aufzufassen sein. Eine Vereinigung mit dem Polkern des anderen Astes findet nicht statt. Hierin stimmen meine Präparate durchaus mit denjenigen von van Tieghem, Treub und Lotsy überein.

Am Eiende dagegen entstehen bald nach der letzten Kernteilung zwei kleine, mit Plasma dicht erfüllte Synergiden und eine meistens etwas größere Eizelle. Vom allgemeinen Schema der Zellgestaltung im achtkernigen Embryosacke der Angiospermen weichen die Zellen des Eiapparates von Balan op h ora in folgenden Einzelheiten ab. Zunächst sind die Größenunterschiede unter denselben nicht so bedeutend, wie es sonst Regel ist. Die Synergiden sind meistens ohne Vakuolen und häufig ist ein Saftraum auch in der Eizelle nicht vorhanden. Der Kern der Eizelle und sein Schwesterkern, der obere Polkern, sind von ähnlicher Größe und Struktur. Der Polkern bleibt in der Nähe der Eizelle liegen. Er nimmt bald an Größe und, nach der Färbung in den Präparaten zu urteilen, auch an Chromatingehalt zu. Bei Verwendung des Flemming'schen Dreifarbengemisches, dessen ich mich, wie Treub und Lotsy, hauptsächlich bediente, nimmt die färbbare Substanz des Polkerns eine intensive Violettfärbung an, die Synergidenkerne werden rot. Dagegen zeigt in meinen Präparaten der Eikern nicht, wie Treub 
und Lotsy in ihren farbigen Zeichnungen angeben, dieselbe intensive Rotfärbung wie die Synergidenkerne. Während diese in meinen Präparaten fast völlig homogen erscheinen, weist der Eikern dicht gehäufte Körner auf und seine Färbung hält ziemlich die Mitte zwischen der Farbe der Synergidenkerne und derjenigen des Polkerns.

\section{Endospermbildung.}

Nach Treub und Lotsy geht die gesamte Weiterentwicklung im Embryosacke von B. elongata und B. globosa vom oberen Polkern aus. Treub speziell hat diesem Nachweis viel Gewicht beigelegt und demselben zahlreiche Figuren gewidmet. Er schreibt (l. c. pag. 15): „Si j'ai représenté dans la planche VI, presque méticuleusement, un aussi grand nombre de stades de la division du noyau polaire, c'est que je tenais, avant tout, à ne plus laisser dans l'esprit du lecteur les moindres doutes sur la vérité de mes assertions, que c'est ce noyau polaire qui se divise - sans union préalable avec un autre noyau polaire - et non l'oosphère, et que c'est lui qui prend l'initiative de tout le développement ultérieur dont le sac embryonnaire devient le théâtre". Meine Nachuntersuchung hat ergeben, daß allerdings die weitere Entwicklung im Embryosacke mit der T'eilungstätigkeit des oberen Polkernes einsetzt, damit aber nur der Grund zur Endospermbildung gelegt ist, während die Embryobildung - aus der Eizelle - erst viel später nachfolgt. Es sei daher der Darstellung der Embryogenese im nachfolgenden auch diejenige der Endospermbildung vorangestellt.

Nachdem sich die Zellen des Eiapparates vom Zytoplasma des Eipoles abgegliedert haben, verbleibt um den Polkern noch eine größere Menge freien Plasmas. Dasselbe nimmt während des nachfolgenden Wachstums des Embryosackes an Menge zu und wird in der Umgebung des Eiapparates schwach vakuolig. Der Polkern wächst ebenfalls, zeigt stark färbbares Chromatin und statt eines einzigen nun häufig zwei bis drei Kernkörperchen (Taf. I, Fig. 13 und 14). Die erste mitotische Teilung des Polkerns findet (Taf. I, Fig. 18), wie auch von Treub beobachtet und dargestellt worden ist, mit in der Längsrichtung des Sackes verlaufender Achse statt. Leider besitze ich auch von dieser Teilung nur Bilder mit seitlicher Ansicht der in der Äquatorialebene liegenden Chromosomen. Habitus und Größe der ganzen Teilungsfigur stimmen aber, wie jedenfalls auch die Chromosomenzahl, mit derjenigen der Embryosackmutterzelle völlig überein. Der ersten Kernteilung folgt sofort eine Zellteilung nach. Sie gliedert den Embryosackraum in 
eine kleinere obere, dem Eiapparat zugekehrte Zelle und eine große Basalzelle, welche den ganzen großen Saftraum und auch den antipodialen Arm des Embryosackes umfaßt. Die beiden Zellen sind zunächst voneinander nur durch Plasmahäute, später auch durch eine konvex nach unten vorgewölbte Wand abgegrenzt. Infolge der kontrahierenden Wirkung des Fixierungsmittels stehen in den Präparaten die Protoplasten der beiden Zellen meistens ziemlich weit vonaneinander ab (Taf. I, Fig. 17 und 18). Die Weiterentwicklung ist, wie Treub richtig erkannt hat, auf die kleinere, vordere Zelle beschränkt. Man kann sie als erste Endospermzelle bezeichnen. Die große Restzelle, die in Analogie zu den Befunden bei zahlreichen anderen Angiospermen als Basal-oder Haustorialzelle funktioniert (vgl. z. B. Ernst und Bernard 1912, pag. 179; Samuelsson 1913, pag. 144) hat keinen Anteil an der Endospermzellbildung.

Der Kern der ersten Endospermzelle ist in der Regel ziemlich zentral gelegen (Taf. I, Fig. 16). Das von zahlreichen kleinen Vakuolen durchsetzte Plasma zeigt sowohl nach der ersten Kernteilung wie auch während der Vorbereitungen zur folgenden Teilung eine radialfaserige Struktur um den Kern. Die nächstfolgenden Entwicklungsstadien des Endosperms scheinen Treub und Lotsy nur in nicht median getroffenen Samenanlagen vorgelegen zu haben. Nach beider Darstellung sollen die weiteren Teilungen ziemlich unregelmäßig verlaufen. Dies ist aber nach meiner Feststellung durchaus nicht der Fall, vielmehr finden wenigstens die drei nachfolgenden Teilungen noch mit großer Regelmäßigkeit in bestimmten Richtungen statt.

Die erste Teilung der Endospermzelle erfolgt in der Regel durch eine Längswand (Taf. I, Fig. 17) ${ }^{\mathbf{1}}$ ). Eine nächstfolgende, in beiden Zellen gleichzeitig und in der Querrichtung eintretende Teilung (Taf. I, Fig. 18 und Taf. II, Fig. 1) erzeugt vier Endospermzellen. Durch eine dritte Teilung wird ein achtzelliger Zellkörper gebildet, dessen Zellen in zwei Etagen zu je vier Zellen angeordnet sind (Taf. II, Fig. 2). Die weiteren Teilungen verlaufen weniger regelmäßig. Die Richtung der Teilungswände variiert und auch der Zeitpunkt der Teilung ist in den einzelnen Zellen verschieden. Neben weiteren Teilungen in der Querrichtung erfolgen auch solche durch perikline Wände, so daß, namentlich in der Umgebung des Embryos, eine größere Zahl von kleineren Endospermzellen entstehen.

1) Die Protoplasten der beiden Tochterzellen sind unter der Einwirkung der Fixierflüssigkeit stark kontrahiert worden. Auch in den folgenden Zeichnungen sind die Kontraktionen des Plasmas vollkommen den Präparaten getreu gezeichnet worden. 
Während dieser Zunahme der Zellenzahl vergrößert sich auch das Volumen der ganzen Endospermzellgruppe. Die Zellen der oberen Etage des Endosperms wachsen, teilweise unter Verdrängung des umgebenden, schon in Auflösung begriffenen Gewebes, um den Eiapparat oder die von diesem allein noch erhaltene Eizelle herum. Die Hauptvergrößerung des Endosperms aber findet basalwärts, auf Kosten der Basalzelle statt, die also immer kleiner wird, später noch in Form einer schmalen Calotte an der Basis des Endospermkörpers vorhanden ist und zuletzt ganz fehlt. In einzelnen Samenanlagen erfährt zwar während des weiteren Verlaufes der Endospermentwicklung auch der Kern der Basalzelle eine Teilung. In diesem Falle kommt es, wie auch Treub und Lotsy bemerken, niemals zu einer nachfolgenden Zellteilung. Die beiden Kerne bleiben in geringem Abstande voneinander (Taf. II, Fig. 7), meistens dicht aneinandergeschmiegt, im Wandbelag der Basalzelle liegen. Die Teilung des Kerns selbst wurde nur in einem einzigen Sacke beobachtet. Sie findet durch Mitose statt.

Hier und da fallen in zweikernigen Basalzellen beträchtliche Unterschiede in Größe und Färbung der Kerne auf. Es scheint mir nicht ausgeschlossen, daB in solchen Fällen die beiden Kerne nicht Teilungsprodukte des einen Kerns sind, sondern daß der erhalten gebliebene zweite Polkern in einer nachträglichen Wanderung den Weg, zwar nicht zum oberen Polkern, aber doch zum Kern der Basalzelle, gefunden hat.

Während der letzten Teilungen der Endospermzellen beginnt in denselben schon die Speicherung von Reservestoffen, vornehmlich Öl und Eiweiß. Das Plasma bildet zwischen den an Zahl und Größe rasch zunehmenden ölhaltigen Vakuolen ein feines Maschennetz (Taf. II, Fig. 10). Der Kern wird dabei ebenfalls mehr und mehr eingeengt und zwischen einigen Vakuolen zu einem pseudopodienartig ausgezogenen, gleichmäßig intensiv gefärbten Körper umgewandelt (Taf. II, Fig. 10). Zwischen diesen Zellen des Endosperms und denjenigen des Embryos sind so weitgehende Unterschiede vorhanden, daß eine Verwechslung völlig ausgeschlossen ist. In bezug auf die definitive Ausbildung des Endosperms sowie Entwicklung und Bau der Samenschale sei wiederum auf die früheren Arbeiten, insbesondere diejenigen von Treub und Van Tieghem, verwiesen.

\section{Embryobildung.}

Über den Ursprung des Embryos von B. elongata gibt Treub bekanntlich an, daß nach dem völligen Verschwinden der Eizellgruppe 
eine zentrale Zelle des Endosperms zur Mutterzelle des Embryos werde. Dieser Ursprung und die weitere Entwicklung werden wie folgt beschrieben (1898, pag. 19):

„Si l'on examine des organes un peu plus âgés, chez lesquels le corps endospermique, provenant de la cellule primaire supérieure de l'endosperme, remplit presque tout le sac embryonnaire, on remarque, du milieu des cellules endospermiques, le plus souvent un peu vers le haut, une cellule ne touchant nulle part à la périphérie, qui se distingue par un protoplasme plus dense.

Cette cellule interne est la cellule-mère du pseudo-embryon. Son origine est fort simple; elle est détachée. d'une des grandes cellules d'endosperme par une cloison parallèle à la surface."

Aus dieser besonderen Zelle entsteht nach Treub's Darstellung durch mehrere aufeinanderfolgende Teilungsschritte ein kleiner Zellkörper. Die größte Zellenzahl, die Treub auf einem einzigen Schnitt durch den Embryo gezählt hat, beträgt fünf und er nimmt an, daß die Zellenzahl der Embryonen zwischen 5 und 10 liegen werde, mit der Einschränkung, daß das erwähnte Maximum wohl nur selten erreicht werde. Nach einigen weiteren Bemerkungen über das Fehlen von Pollenschläuchen in den weiblichen Organen schließt er seine Ausführungen über die Entstehung des Embryos mit dem Satze (1. c. pag. 21):

„L'exposé qu'on vient de lire aura donné, je l'espère, la conviction qu'il n'est pas question ici de parthénogénèse, mais que c'est du noyau polaire, frère du noyau de l'oosphère, que relève toute la seconde phase du développement du sac embronnaire.

Le fait que le pseudo-embryon se montre seulement sur le tard et la manière dont il prend naissance viennent corroborer, au surplus, la conclusion que l'oosphère avorte."

Nach Lotsy liegen bei B. globosa ganz und gar dieselben Verhältnisse wie bei B. elongata vor. Auch er gibt an, daß der Embryo apogam aus dem als Prothallium zu deutenden Teilungsprodukte des Eipolkernes hervorgehe.

Dieser völligen Übereinstimmung der durch zwei Forscher an zwei verschiedenen Arten erhaltenen Resultate kommt nun aber nicht diejenige Beweiskraft $\mathrm{zu}$, die $\mathrm{ihr}$ vielfach zugeteilt worden sein mag. Man hat wohl häufig übersehen, daß die Arbeit Lotsy's keinen Anspruch auf kritische Nachuntersuchung macht, sondern als kürzere Parallelarbeit aufgefaßt sein will, von der für den Verfasser von vornherein feststand, daß sie zu einer Bestätigung der Treub'schen 
Angaben führen werde. Dies geht wohl am besten aus Lotsy's eigenen Worten hervor, wenn er in seiner Einleitung (1899, pag. 174) schreibt: „Trotzdem die Arbeit Treub's, mit den beigegebenen schönen Tafeln, auch nicht den geringsten Zweifel an dem bei seiner Art gefundenen Verhalten zuläßt, schien es mir doch nicht ohne Interesse, eine dritte Art zu untersuchen." Auch gegen den Schluß der Arbeit hin bemerkt er wieder (l. c. pag. 184): „Ich habe es dieser großen Übereinstimmung wegen denn auch nicht für nötig erachtet, so viele Stadien abzubilden oder die Sache so minutiös zu untersuchen und zu beschreiben, wie solches von Treub geschehen ist."

Meine eigene Untersuchung ist nun zwar zum großen Teil an Material von B. elongata ausgeführt worden; da aber gerade eine Anzahl der entscheidenden Stadien dem Material von B globosa entstammen, so steht wohl sicher, daß beide Arten nicht nur in den schon von Treub und Lotsy richtig aufgefaßten Entwicklungsvorgängen, sondern auch in denjenigen der Endosperm- und Embryoentwicklung übereinstimmen. Ich gehe also dazu über, meine eigenen Beobachtungen über die Embryobildung von Balanophora elongata und B. globosa wiederzugeben und hernach $\mathrm{zu}$ untersuchen, welche Umstände wohl $\mathrm{zu}$ der irrtümlichen Darstellung eines speziell um die Embryologie der Angiospermen so hoch verdienten Forschers, wie es M. Treub gewesen ist, Anlaß gegeben haben mögen.

Bei der embryologischen Untersuchung einiger javanischer Burmannia-Arten hatte ich, wie schon einleitend bemerkt worden ist, festgestellt, daß bei den befruchtungsbedürftigen Arten (B. Championii und B. candida), wie bei der parthenogenetischen B. coelestis. die Entwicklung des Embryos derjenigen des Endosperms erst sehr spät nachfolgt. $\mathrm{Zu}$ einer Zeit, da schon der ganze Embryosackraum mit Endospermgewebe angefüllt ist und die Speicherung von Reservestoffen in dessen Zellen einsetzt, findet sich am Scheitel des Nährgewebes immer noch eine ungeteilte Keimzelle vor. Ihr Volumen ist bedeutend geringer als dasjenige der Eizelle kurz nach erfolgter Zellbildung im achtkernigen Embrysacke. Das rührt davon her, daß bei B. Championii und B. candida nach der Befruchtung, bei der parthenogenetischen B. coelestis zu Beginn der Endospermbildung, offenbar eine Kontraktion des Ei-Inhaltes stattfindet. Der Saftraum verschwindet, der Zellraum wird dadurch erheblich verkleinert und ist durch Zytoplasma und Kern völlig ausgefüllt. Da während dieser Gestalts- und Größenveränderung der Keimzelle ihre Empfindlichkeit gegen Einwirkung von Fixierungsmitteln offenbar besonders groß ist, erweckt sie in den Prä- 
paraten häufig den Eindruck, als sei sie mit den Synergiden in Degeneration begriffen. $\mathrm{Zu}$ ganz gleichen Ergebnissen führten die Untersuchungen an Thismia-Arten und wie schon pag. 132 angeführt wurde, auch bei Sciaphila und Cotylanthera. Die auffallendste Verkleinerung erfährt die Eizelle während der Umgestaltung zur Keimzelle bei Cotylanthera. Hier ist, wie ich einer späteren, ausführlichen Darstellung der Verhältnisse vorgreifend bemerken möchte, zur Zeit der freien Endospermkernteilung die Eizelle so klein, daß sie nur noch aus dem Zellkern und einer denselben umhüllenden dünnen Plasmaschicht besteht und infolge der geringen. Größe und der Lagerung am basalen Pol des Embryosackes erst nach langem Suchen entdeckt worden ist.

Ganz ähnlich liegen nun die Dinge auch bei Balanophora. Die Bilder, die der Eipol des Embryosackes während der ersten Stadien der Endospermbildung bietet, täuschen noch um so mehr eine völlige Degeneration des ganzen Eiapparates vor, als, wie auch Treub schreibt, dieser Teil des Embryosackes nur von einer dünnen Membran umgeben ist und auch deshalb bei der Präparation leicht Schrumpfungen erfährt. Seine Zellen erhalten dadurch ein ähnliches Aussehen wie die an der Oberfläche, speziell am Scheitel des Embryosackes, in Auflösung begriffenen Nuzelluszellen.

Aufgelöst werden aber nach meiner Feststellung zu Beginn der Endospermbildung nur eine oder auch beide Synergiden nebst dem umgebenden Nuzellusgewebe. Über der ersten Endospermzelle findet man daher in einer Gruppe vereinigt die degenerierenden Synergiden und die verjüngte und infolge der Präparation leicht geschrumpfte Eizelle (Taf. I, Fig. 16). Die Synergidenkerne sind gleichmäßig rot gefärbt, ohne Kernkörperchen und häufig von unregelmäßigen Umrissen. Öfters kommt es auch zu einem Zerfall ihrer Kerne in zwei bis vier unregelmäßig geformte Stücke, die nebeneinander oder an verschiedenen Stellen der kontrahierten Zellen liegen. Bei der undeutlichen Abgrenzung der Synergidenzellen kann infolge dieser Kernfragmentation leicht der Eindruck entstehen, als seien hier Reste von drei oder mehr degenerierenden Zellen vorhanden. Neben den immer kleiner werdenden Resten der Synergiden liegt die ebenfalls deformierte Eizelle, die aber deutlich strukturiertes Plasma und einen chromatinreichen Kern enthält. In diesem Zustande verharrt sie auch während der nachfolgenden Stadien der Endospermbildung (Taf. I, Fig. 17 und 18). Da die den Embryosackscheitel bedeckenden Zellen in Auflösung begriffen sind, ist es begreiflich, daß das Endosperm sein Wachstum auch nach oben ausdehnt und dabei die Eizelle seitlich umwächst. Die Wahrnehmung 
der Eizelle wird dadurch noch mehr erschwert und nur äußerst selten trifft man bei der Durchmusterung von Präparaten mit solchen Entwicklungsstadien auf Schnitte, in welchen (Taf. II, Fig. 2) die Keimzelle durch die bedeckende, inhaltsreiche Endospermzelle hindurch sichtbar ist. Nun erst setzt die eigentliche Keimentwicklung ein. Die Keimzelle zeigt reichlicheres und stärker färbbares Plasma und beginnt sich unter gleichzeitiger Größenzunahme des Kernes in die Länge zu strecken. Während dieses Wachstums bilden sich in ihrem Plasma eine, zwei oder mehrere Vakuolen (Taf. II, Fig. 3). Durch eine erste Kernteilung entstehen zwei hintereinander liegende Kerne (Taf. II, Fig. 6 und 7). Hierauf folgt eine Querteilung der Zelle (Taf. II, Fig. 8).

Häufig trifft man an Stelle eines quergeteilten, zweizelligen Embryos zwei nebeneinander liegende, gleichgeformte und mit gleichem Inhalt versehene Zellen (Taf. II, Fig. 4 und 5; vgl. auch Taf. XXXV, Fig. 29 bei Lotsy). Trotzdem ich in keinem Falle eine Kernteilung mit quergestellter Achse oder sonstige Anzeichen dafür gefunden habe, daß diese beiden Zellen durch Längsteilung aus der gestreckten Keimzelle hervorgegangen sind, will ich die Möglichkeit dieser Teilung nicht außer Frage setzen. Wahrscheinlicher allerdings erscheint mir die Möglichkeit, daß es sich in diesen Fällen um Weiterentwicklung von zwei Zellen des Eiapparates handelt. Damit wäre auch in Einklang zu bringen, daß in jüngeren Stadien statt einer einzigen, zuweilen zwei Zellen des Eiapparates ziemlich gut erhalten sind. Ferner spricht dafür der Umstand, daß in noch etwas älteren Stadien an Stelle der zwei nebeneinander liegenden einkernigen Zellen, zwei solche mit zwei oder sogar mit vier Kernen vorhanden sind. Alles das deutet mehr auf eine Weiterentwicklung von zwei ursprünglich getrennten Zellen, als auf eine Längsteilung der einen Keimzelle hin. Ich halte also dafür, daß bei Balanophora elongata und globosa ein ähnliches Verhalten vorliegt, wie es für Burmannia coelestis (Ernst und Bernhard 1912, pag. 248) beschrieben worden ist: Parthenogenesis mit gelegentlicher Polyembryonie.

In den Präparaten Treub's scheinen ähnliche Bilder gefehlt $\mathrm{zu}$ haben. Sie wären ihm sonst gewiß aufgefallen, um so mehr, als Polyembryonie mit seiner Annahme der apogamen Embryobildung aus Prothalliumzellen trefflich harmoniert hätte und eigentlich a priori hätte erwartet werden müssen. Auch Lotsy macht über das Vorkommen von zwei Embryonen in einem Endospermkörper keine Mitteilung (vgl. aber seine Taf. XXIX, Fig. 35), während er über das 
Vorkommen von zwei Embryosäcken mit je einem Embryo in einem fast reifen Samen zu berichten weiß (l. c. pag. 180, Taf. XXIX, Fig. 39).

Die Lage des Embryos im Endosperm ist in jüngeren wie in älteren Samenanlagen erst nach Durchsicht zahlreicher Präparate zu übersehen. In der großen Mehrzahl der Schnitte erscheinen die Embryonen, wie von Treub und Lotsy dargestellt worden ist, rings vom Endospermkörper umschlossen und, wie Treub schreibt, dem vorderen Ende desselben etwas genähert (Taf. II, Fig. 9). Nach langem Suchen sind aber eine ganze Anzahl Samenanlagen gefunden worden, in welchen der Embryo bis an die Oberfläche des Endosperms reicht. Einige dieser Stadien sind in den Figuren 4, 7 und 8 (Taf. II) dargestellt worden. Auch in vielen Schnitten mit rings von Endosperm umschlossenen Embryonen läßt die Anordnung der Endospermzellen erkennen, daß es sich entweder um tangential geführte Schnitte oder um eine nachträgliche Umwachsung einer ursprünglich an der Oberfläche liegenden Zelle handelt.

Von den durch die erste Querteilung entstandenen zwei Zellen des Proembryos wird die eine zur Suspensorzelle. Die Weiterentwicklung des Embryos geht von der scheitelständigen Zelle aus. Weiter entwickelte Stadien wurden nur wenige gefunden. Häufig sind nur noch Stadien; in welchen die Endzelle zwei oder vier Kerne enthält oder in ebensoviele Zellen zerlegt worden ist. Der einzige, bedeutend weiter entwickelte Embryo ist in Taf. II, Fig. $11 a$ und $b$, dargestellt. Außer einer schmalen, bis an die Oberfläche des Embryosackes reichenden Stielzelle weist derselbe drei zweizellige Etagen auf. Die Stielzelle dieses Embryos ist von den Etagenzellen durch geringeren Plasmagehalt und das Fehlen des Kerns unterschieden. Sie ist also schon in Rückbildung begriffen, dabei allerdings erst so wenig degeneriert, daß sie noch absolut sicher als Bestandteil des Embryos erkennbar ist. Es ist sehr wohl möglich, daß in anderen Samenanlagen die Auflösung der Basalzelle früher und rascher vor sich geht, ihr Platz von den benachbarten Endospermzellen eingenommen wird und so der Keimling völlig ins Innere des Endosperms zu liegen kommt.

Es sind also, wenn wir den Entwicklungsgang der zehr kleinen und wenig differenzierten Embryonen von Balanophora überblicken, drei verschiedene Umstände, deren Zusammenwirken die Feststellung obiger Tatsachen erschweren und Treub bei seiner Darstellung der Embryoentwicklung auf eine falsche Bahn drängten: 
1. Die kontrahierende Wirkung der Präparationsflüssigkeiten, die sich auf den ganzen Embryosackinhalt, besonders aber auf die Elemente des Eipoles geltend macht ${ }^{1}$ ).

2. Die Volumen- und Formänderung der Eizelle zu Beginn der Weiterentwicklung des Embryosackinhaltes, welche zusammen mit der Wirkung der Fixierflüssigkeit leicht eine Degeneration des ganzen Eiapparates vortäuscht.

3. Die auffallend verspätete Embryoentwicklung. Wachstum und Teilung der Keimzelle erfolgen zu einer Zeit, da durch den dichten Inhalt der Endospermzellen und deren Wachstum die Auffindung der Eizelle erschwert ist. Bei der Kleinheit der Objekte und ihrer ungünstigen Lagerung ist daher die Gewinnung entscheidender Bilder vom Zufall oder sehr langem Suchen abhängig.

Nach unserer Feststellung geht also bei Bal anophora elongata und B. globosa der Embryo aus der Eizelle des Eiapparates hervor. Es bleibt also noch zu entscheiden, ob dieser Embryobildung auch eine Befruchtung vorausgeht oder nicht. Hierin möchte ich mich, allerdings

1) Von dem zur Verfügung stehenden Untersuchungsmaterial sind hauptsächlich die von mir mit $96 \%$ Alkohol, sowie die von Prof. Senn und Dr. Bernard mit absolutem Alkohol fixierten Blütenstände geschnitten worden. Die kontrahierende Wirkung des Alkohols hat sich in den verschiedenen Entwicklungsstadien in verschiedenem Maße geltend gemacht. Fast in allen jüngeren Embryosäcken hat sich der dünne Plasmabelag, der die U-förmige Embryosackhöhle auskleidet, von der Wand abgehoben und liegt als faltiger Schlauch im Zellraume (Taf. I, Fig. 9-12). In achtkernigen Embryosäcken mit Eizelle und Synergiden ist durch die Wirkung der Fixierflüssigkeit eine deutliche Sonderung des dem Polkern zugehörigen Plasmas vom Eiapparat eingetreten. Im Eiapparat selbst weist der Inhalt einer jeden Zelle, auch derjenige der Eizelle, wieder Schrumpfungserscheinungen auf. Indessen ist doch auf diesen Stadien, wie auch später, nach Bildung der ersten Endospermzellen stets erkennbar, daß es sich nicht nur um Schrumpfungen im Eiapparat, sondern um ebenso starke Schrumpfungen im übrigen Embryosackinhalte handelt. So geht also schon aus der Form der Eizelle und des zwischen Eizelle und Polkernplasma, resp. zwischen Eizelle und Endospermzellen entstandenen Kontraktionsraumes in den möglichst getreu nach den Präparaten gezeichneten Figuren 12-14 hervor, daß nicht etwa das Plasma des Embryosackes den Eiapparat zusammenpreßt, wie Treub (l. c. pag. 14) angenommen hat, sondern vielmehrder Eiapparat, resp. dessen Eizellevor der Fixierung stark in das Embryosackplasma vorgewölbt gewesen sein muß. Unter Berücksichtigung der Schrumpfungserscheinungen kann man also an den Bildern noch recht gut die ursprüngliche Größe und Form der Ei- und Keimzelle auf den verschiedenen Entwicklungsstadien bestimmen. Es sei noch darauf hingewiesen, $\mathrm{da} ß$ in dem von Treub und Lotsy untersuchten Material, nach ihren Zeichnungen zu schließen, eben solche, wenn nicht noch stärkere Kontraktionen in den Embryosäcken eingetreten waren.

Flora, Bd. 106. 
wiederum nicht ohne Einschränkung, der Ansicht von Treub und Lotsy anschließen.

Tr e ub erwähnt (1. c. pag. 20), daß er an den weiblichen Organen von B. elongata niemals einen Pollenschlauch gesehen habe. Ich fand trotz eingehenden Suchens ebenfalls keine keimenden Pollenkörner, doch sind in einem meiner Präparate drei Samenanlagen enthalten, von denen die eine im griffelähnlichen Fortsatz von einem Pollenschlauch durchwachsen $\mathrm{zu}$ sein scheint, während in den beiden anderen ein Pollenschlauchende am oberen Rande des Eiapparates vorhanden sein könnte. Natürlich möchte ich diesen drei Fällen kein entscheidendes Gewicht beilegen, doch ist damit die Möglichkeit angedeutet, daß bei Balanophora elongata neben vorwiegend parthenogenetischer Embryobildung gelegentlich auch die eine oder andere Eizelle befruchtet wird. Es könnte sich also B. el on gata denjenigen parthenogenetischen Pflanzen anschließen, bei denen, wie zuerst für Thalictrum purpurascens gezeigt worden ist, gelegentlich noch Chromosomenreduktion und Befruchtung erfolgen können. Hierfür speziell bei Balanophora aber den Nachweis zu erbringen, dürfte in Anbetracht all der Schwierigkeiten, die sich der Untersuchung entgegenstellen, nur durch einen dem erreichbaren Resultat keineswegs entsprechenden Zeitaufwand möglich werden. Für Bala n oph ora gl ob o sa liegen die Verhältnisse wesentlich günstiger. Für diese Spezies ist auf Grund der Lotsy'schen Untersuchung und meines Nachweises der Entstehung des Embryos aus der Eizelle somatische Parthenogenesis wohl sicher. Schon in der Überschrift seiner Mitteilung bezeichnet Lotsy Balanophora globosa als eine „örtlich verwitwete Pflanze“. Männliche Pflanzen waren an seinem Fundorte, dem Pengalenganplateau auf Java, nicht vorhanden. Außerdem hat Lotsy völlig einwandfrei durch das Experiment den Nachweis erbracht, daß bei dieser Balanophora-Art Samenbildung ohne Befruchtung erfolgt. Er konnte zeigen, daß weibliche Pflanzen auch an isoliert entstandenen Blütenständen die charakteristischen Samen mit Endosperm und Embryo erzeugten (l. c. p. 177).

\section{Vergleich mit den Ergebnissen embryologischer Unter- suchungen an anderen Balanophoraceen.}

Schon einige Jahrzehnte vor Treub und Lotsy haben mehrere Botaniker die Fortpflanzungsvorgänge bei Balanophora und anderen Vertretern der Balanophoraceen studiert. Der Arbeit Treub's war ferner eine solche von Van Tieghem über B. indica vorausgegangen. Sowohl Van Tieghem wie Treub stellen ihre Befunde in starken 
Gegensatz zu den Ergebnissen der früheren Autoren. Nach Treub ist alles was bis 1896 über die weiblichen Blüten von Balanophora in die Lehrbücher übergegangen ist und zum größten Teil auf den Altmeister der pflanzlichen Embryologie, H of m e is ter, zurückgeht (1858, pag. 110 und 1859, pag. 585) durch und durch unrichtig. „Il se trouve que tout ce qui a été publié par lui (Hofmeister), sur l'organe femelle du Balanophora, est erroné, et cela à tel point qu'on ne réussit pas à se rendre compte, comment un investigateur d'un aussi grand mérite, ait pu commettre de si graves erreurs (l. c. pag. 1).

Bei der Redaktion seiner Abhandlung hatte Treub die etwa ein Jahr zuvor erschienene Arbeit von Van Tieghem vorgelegen und er gibt in seiner Einleitung die B. indica betreffenden Ausführungen Van Tieghem's in extenso wieder. Seine eigenen Befunde stimmen mit denjenigen Van Tieghem's, soweit sie die Entwicklung des weiblichen Organs selbst, sowie die Entwicklung des Embryosackes bis zum achtkernigen Stadium betreffen, in den Hauptzügen überein. In den Details allerdings weichen die beiden Darstellungen häufig voneinander ab. Auf Grund seiner viel einläßlicheren Untersuchung kann Treub verschiedene Angaben Van Tieghem's, wie z. B. diejenige, daß der Plasmaschlauch des Embryosackes allein U-Form habe, die Zellulosewand in Beziehung zur Achse dagegen symmetrisch bleibe, sicher widerlegen. Nach Abschluß der Zellbildung im achtkernigen Embryosacke konstatierte Van Tieghem bei Balanophora indica ebenfalls, daß die Verschmelzung der beiden Polkerne ausbleibt und die Entwicklung des Endosperms vom oberen Polkern allein ausgeht. In starkem Widerspruch zu Treub stehen aber seine Angaben über den Ursprung des Embryo. Er gibt für den Embryosack von B. indica das Vorkommen eines völlig normal entwickelten Eiapparates an und stellt fest (1896, pag. 306), daß dessen Eizelle durch einen Pollenschlauch befruchtet wird. Treub selbst hat den Widerspruch zwischen seinen eigenen Angaben über die Entstehung des Embryos und denjenigen Van Tieghem's nicht diskutiert, sondern sich mit dem, wie ihm schien, einwandfreien Nachweis der apogamen Embryobildung bei der von ihm untersuchten Art begnügt. Einer Verallgemeinerung der Resultate weniger abgeneigt, schrieb dagegen Lotsy in seiner Schlußbetrachtung (l. c. pag. 184):

„Mir scheinen die hier beobachteten Tatsachen zur Genüge zu zeigen, daß B. globosa in ihrer Entwicklung vollständig mit B. el on ga ta übereinstimmt, so daß die von Tre ub für B. elong a ta angegebene Entwicklung wohl für das ganze Genus Balanophora gelten wird. 
Die von Van Tieghem an Balanophora indica erhaltenen Resultate werden wohl aus dem ungenügenden Zustande des diesem großen Forscher zurVerfügung stehenden Materials erklärt werden müssen."

In einer der Untersuchung über Balanophora bald nachfolgenden Studie über Rhopalocnemis phalloides stellt Lotsy (1901) fest, daß bei diesem Vertreter der Balanophoraceen der Embryo aus der Eizelle hervorgeht. Dieser Ursprung des Embryos wird auch durch Zeichnungen belegt. Von einer derselben (Taf. XI, Fig. 54) sagt er, daß sie ,puts it beyond the shadow of a doubt that the embryo is, formed from the egg-cell“. Von einer anderen Figur (Taf. XI, Fig. 53) welche einen Endospermkörper mit ringsumschlossenem Embryo darstellt, wird dagegen geschrieben (1. c. pag. 90), daß sie „,might give rise to the idea that the embryo was formed apogametically as in Balanophora; this dilusion is caused, by the fact that the section is not a median one in regard to the eggcell and thus has not touched the stalk of the eggcell".

Ob Parthenogenesis vorliegt oder Befruchtung eintritt, konnte Lotsy für Rhopalocnemis nicht absolut sicher feststellen. Doch erscheint ihm Parthenogenesis wahrscheinlicher.

Auffallend ist, daß Lotsy bei diesem Befund sich nicht seiner früheren Ablehnung der Angaben Van Tieghem's und seiner eigenen Verallgemeinerung der Treub'schen Ergebnisse für die ganze Gattung Balanophora erinnert hat. Es scheint, als hätte er durch seinen eigenen Nachweis der wahrscheinlich parthenogenetischen, sicher aber aus der Eizelle stattfindenden Embryobildung bei einer Balanophora sehr nahe stehenden Gattung zu einer Revision der Angaben über Balanophora geradezu herausgefordert werden müssen. Merkwürdigerweise ist dies nicht der Fall gewesen, denn er schreibt auf derselben Seite weiter:

"The fact which has been absolutely proved and which is not without interest is that in the Balanophoraceous family the embryo may be formed in two very different ways viz.: among Balanophora elongata (Treub) and Balanophoraglobosa (Lotsy) apogamically from endospermic cells, among Rhopalocnemis phalloides from the egg cell."

Nun, zwei Wege der Embryobildung sind auch nach meiner Meinung innerhalb der Balanophoraceen zu unterscheiden, aber beide nehmen ihren Ausgang von der Eizelle. In einem Falle findet parthenogenetische Entwicklung derselben statt, so bei B. globosa, sehr wahrscheinlich auch bei B. elongata und Rhopalocnemis 
phalloides, vielleicht auch bei Arten anderer Gattungen der Familie. Bei anderen Vertretern der Balanophoraceen geht der Embryobildung dagegen eine normale Befruchtung der Eizelle voraus. Der Beweis hiefür scheint mir in der bereits vorliegenden Literatur gegeben $\mathrm{zu}$ sein. Zunächst ist zu erwähnen, daß Van Tieghem in einer neueren Arbeit (1907, pag. 174) in der Lage war, nicht nur seine früheren Angaben über $B$. indica aufrecht zu erhalten, sondern auch durch Untersuchungen an zwei weiteren Balanophora-Arten, B. dentata und B. pentamera zu ergänzen. Die Vorgänge der Endosperm- und Embryobildung hat Van Tieghem dabei leider nicht selbst untersucht. Er bezieht sich in seiner zusammenfassenden Darstellung auf die Ergebnisse der älteren Autoren, speziell auf Hofmeister. Dabei hebt er hervor, daß er selbst gezwungen gewesen sei, die großen Irrtümer aufzudecken, die in dessen Darstellung der Entwicklung des weiblichen Organs von Balanophora bis zur Eibildung enthalten sind und daß die Treub'sche Darstellung die Richtigkeit seiner Korrekturen vollauf bestätigt habe. Trotzdem stimmt er aber dem vernichtenden Urteil Treub's über die Hofmeister'sche Untersuchung nicht zu und weist, wie sich jetzt zeigt mit Recht, darauf hin, daß Unrichtigkeiten im ersten Teil der Darstellung Hofmeister's die Richtigkeit der Angaben im zweiten Teil nicht ausschließen.

Er schreibt: „Malgré la grave erreur qu'il a commise dans la structure du carpelle avant la formation de l'oeuf, en lui attribuant un ovule qui n'existe pas, cette seconde partie des résultats de Hofmeister, avec les figures qui les accompagnent et les appuient, me parait mériter pleine confiance. Il serait injuste, à mon avis, de les regarder tous, à cause de cette faute initiale, comme nuls et non avenus, en creusant ainsi du même coup une grande lacune dans nos connaissances. Il ne serait pas légitime non plus de chercher ensuite à combler cette lacune indirectement, en étendant purement et simplement au genre Balanophore les faits probablement exceptionels qui ont été observés plus récemment dans deux espèces de Java." Sein Wunsch, es möchten bald neue Untersuchungen die Richtigkeit der Hofmeister'schen Resultate prüfen, scheint durch meine Untersuchung bereits erfüllt und seine Vermutung, daß die Angaben Hofmeister's über Embryo- und Endospermbildung nicht ohne weiteres abgelehnt werden dürfen, sondern Vertrauen verdienen, hat sich vollkommen richtig erwiesen.

Der Vergleich der Hofmeisterschen Figuren (1859, Taf. XV, Fig. 8-13) mit den meinigen ergibt für den zweiten Teil seiner Untersuchung eine geradezu glänzende Rechtfertigung. Zieht man auch den 
Text zum Vergleich heran, so zeigt sich eine weitgehende Übereinstimmung in Endosperm- und Embryobildung bei B. elongata und B. globosa einerseits und den von Hofmeister untersuchten B. polyandra, fungosa, B. dioica andererseits. Der einzige Unterschied besteht darin, daß bei B. el on gata und globosa der Embryo sehr wahrscheinlich aus der unbefruchteten, bei den von Hofmeister untersuchten Arten aus der befruchteten Eizelle hervorgeht. Ich lasse Hofmeister's Angaben über die Embryogenese unter Weglassung einiger weniger wichtiger Details (l. c. pag. 589) nachfolgen:

„Mehrmals wurde bei Balanophora polyandra und fungosa eine fädliche Zelle, mit das Licht stark brechendem Inhalte, in Berührung mit dem oberen Teile des Embryosackes beobachtet. Kein Zweifel, daß sie das Ende des Pollenschlauches ist. Überall, wo sie wahrgenommen wurde, war nur noch eines der Keimbläschen vorhanden, und der ganze Raum des Embryosackes in mindestens zwei Tochterzellen geteilt. Diese zwei ersten Zellen des Endosperms werden durch das Auftreten einer Längsscheidewand gebildet. Dieser ersten Teilung folgt sofort die durch auf der zuvorgebildeten rechtwinkelige Längs-, dieser die durch Querwände. In den acht Zellen des Endospermkörpers treten darauf verschieden geneigte Wandungen auf."

Diese Darstellung stimmt, wie man sieht, vollständig mit meiner Beschreibung der Entwicklung des achtzelligen Endospermkörpers aus der nach der ersten Teilung des oberen Polkerns entstandenen Endospermzelle überein. Der einzige Irrtum Hofmeister's in bezug auf die Endospermbildung beruht darin, daß er den ersten zur Endospermbildung notwendigen Teilungsschritt übersehen hat und die Zelle, „deren ganzer Raum durch eine Längswand in zwei Tochterzellen geteilt wird", eben nicht der Embryosackraum, sondern bereits eine erste Endospermzelle ist.

Bei der Feststellung der Embryoentwicklung verzeichnete Hofmeister ebenfalls die Wahrnehmung, daß das befruchtete Keimbläschen sich zunächst nicht wesentlich verändert und sich während der Endospermbildung kaum merklich in die Länge streckt. Die weitere Entwicklung des Embryos wird (1859, pag. 591) wie folgt geschildert:

„Im heranwachsenden Endosperm verlängert sich das befruchtete Keimbläschen zu einem bis nahe an den Mittelpunkt des Zellenkörpers wachsenden Embryonalschlauche, dicht über dessen Ende dann eine Querwand entsteht. Bisweilen, doch nicht immer, wiederholt sich diese Querwandbildung in der unteren Zelle des zweizelligen Vorkeims. Die Umbildung seiner Endzelle zum Embryokügelchen wurde nur in solchen 
Früchten beobachtet, in denen das Endosperm die Fruchtknotenhöhle (soll heißen den Embryosack) völlig ausfüllte und die alle Zeichen der vollständigen Reife trugen. Hier erschien die Endzelle des Vorkeims durch übers Kreuz gestellte Längsscheidewände in vier Zellen geteilt.“

Auch in dieser sich in der Hauptsache auf B. dioica beziehenden Darstellung der Embryobildung ist also eine weitgehende Übereinstimmung mit meinen Angaben (vgl. pag. 144) nicht $\mathrm{zu}$ verkennen. Für die Beurteilung dieser Übereinstimmung dürfte die Erklärung wertvoll sein, daß ich während meiner ganzen Untersuchung und auch bei der Ausführung meiner Zeichnungen nur die Arbeiten von Treub und Lotsy vor mir hatte. Um so überraschender war es dann für mich selbst, bei der während der Ausarbeitung des Manuskripts erfolgten Durchsicht der älteren Literatur zu ersehen, daß die von Hofmeister und mir bei der Untersuchung verschiedener Arten erhaltenen Resultate eine gegenseitige Bestätigung bilden.

Nachdem also gezeigt worden ist, daß die Hofmeister'sche Darstellung der Embryo- und Endospermbildung bei den von ihm untersuchten Balanophora-Arten vollen Anspruch auf Richtigkeit machen darf, wird man auch seine Angaben über das Anhaften von Pollenkörnern an der Spitze des griffelartigen Fortsatzes, über Pollenschlauchbildung und Wachstum des Pollenschlauches bis zum oberen Ende des Embryosackes nicht länger übersehen dürfen. Noch um so weniger, als sich gerade in diesen Punkten Hofmeister auf ähnliche Beobachtungen von Griffith (Transact. Linn. Soc. 20, pag. 99 und Taf. VII, Fig. 13 und 16) und Hooker (Transact. Linn. Soc. 25, Taf. V, Fig. 15-17) beziehen konnte. Man wird nun ferner auch seinen Angaben über andere Balanophoraceen (z. B. Langsdorffia hypogaea Mart. 1859, pag. 578, Sarcophyte sanguinea Sparrm. 1859, pag. 583, Phyllocoryne jamaicensis Hook. 1859, pag. 598) hinsichtlich Bestäubung, Befruchtung, Embryo- und Endospermbildung Glauben schenken müssen. Ziehen wir also auch diese Angaben Hofmeisters, sowie die auch von van Tieghem berücksichtigten Ergebnisse von Griffith, Hooker und anderen älteren Autoren zum Vergleich heran, so ergibt sich, daß bei allen bis jetzt untersuchten Balanophoraceen der Embryo aus der Eizelle hervorgeht und auch in späteren Stadien noch häufig durch einen Träger bis an die Embryosackwand reicht.

Bei Balanophora polyandra, fungosa, volucrata, B. indica, bei Cynomorium coccineum, Langsdorffia hypogaea, Sarcophyte sanguinea, Phyllocoryne jamaicensis sind entweder Pollen- 
körner auf der Narbe, Pollenschläuche im Griffel oder am Eiapparat festgestellt worden. Damit ist für diese Vertreter der Balanophoraceen die Befruchtung zwar nicht völlig erwiesen, Embryobildung aus der befruchteten Eizelle aber immerhin zu erwarten. Für Balanophora elongata, B. globosa, Rhopalocnemis phalloides und Helosis guyanensis Rich. erfolgt die Embryobildung ebenfalls aus der Eizelle, und zwar - was allerdings nicht für alle vier Pflanzen in gleichem Maße wahrscheinlich gemacht worden ist - auf parthenogenetischem Wege.

Einer besonderen Erwähnung bedarf noch die Helosis guy an ensis, über welche nicht nur die älteren Angaben Hof meis ter's (1859, pag. 593, Taf. XVI, Fig. 1-5), sondern auch eine neuere embryologisch-zytologische Untersuchung von Chodat und Bernard vorliegt. In der Literatur wird Helosis guyanensis vielfach (z. B. Coulter und Chamberlain, pag. 218, Winkler 1908, pag. 357 und 1913, pag. 266) auf Grund der Untersuchungsergebnisse der genannten Autoren mit B. globosa und elongata als Beispiel für dieselbe Art der apogamen Embryobildung angeführt. Es wird also vor allem notwendig sein, den Standpunkt der beiden Autoren selbst kennen zu lernen. Auf die von Balanophora stark abweichende Entwicklung des Embryosackes von Helosis (s. Chodat und Bernard, 1900, pag. 76) braucht dabei nicht eingetreten zu werden. In Übereinstimmung mit B. elongata und B. globosa erfolgt auch bei Helosis guyanensis die Endospermbildung aus dem einen, oberen Polkern. Ein Embryo wurde erst in späteren Entwicklungsstadien, scheinbar ohne Suspensor, dem Endosperm eingesenkt gefunden. Er bleibt nach der Darstellung von Chodat und Bernard sehr rudimentär und setzt sich nur aus einer kleinen Anzahl von Zellen zusammen', die einen ungefähr keulenförmigen, von einer Querfurche durchzogenen Körper bilden. Die ersten Stadien der Embryoentwicklung wurden nicht aufgefunden, die beiden Autoren äußern sich daher auch nur äußerst vorsichtig über die Herkunft des Embryos (l. c. pag. 13):

„Faut-il considérer l'embryon d'Helosis comme le produit de l'oeuf fécondé? Vaut-il mieux admettre que, comme chez Balanophora elongata étudié par M. Treub, il est adventif? Nous ne sommes pas en mesure de donner à ces questions une solution définitive.

Il nous semble néanmoins probable que la seconde alternation est la vraie. L'oeuf, au moment où, chez les autres végétaux normalement fécondés, il entre en division et s'allonge pour constituer le suspenseur 
et l'embryon, subit au contraire ici un remarquable appauvrissement et finit par devenir méconnaissable.

La position de l'embryon isolé dans l'endosperme paraît un second indice important permettant de supposer que son origine est endospermique. Si cette supposition se trouve fortifiée par des recherches complémentaires, Helosis guyanensis fournirait un nouvel exemple d'apogamie."

Ich glaube nicht, daß eine Nachuntersuchung dieses Ergebnis zeitigen würde. In Analogie zu den Befunden an Balanophora und den eingangs erwähnten saprophytischen Burmannia-, Sciaphila- und Cotylanthera-Arten würde sich wahrscheinlich zeigen, daß die Eizelle von Helosis guyanensis nur scheinbar degeneriert und einem ersten Stadium der Rückbildung im späteren Verlauf der Endospermbildung ein verspätetes Wachstum nachfolgt. Auch der Umstand, daß der Embryo inmitten des Endospermkörpers gefunden worden ist, wird eine einfachere Erklärung finden, wahrscheinlich dieselbe, die Lotsy selbst für Rhopalocnemis gegeben hat. Selbstverständlich vermögen diese Analogieschlüsse die direkte Feststellung nicht $\mathrm{zu}$ ersetzen. Diese ist aber schon durch $\mathrm{H}$ of meister erfolgt. Auch in seiner Darstellung der Entwicklungsvorgänge von Helosis werden, trotz nachgewiesener großer Fehler nicht alle Angaben in Zweifel gezogen werden dürfen und wenn er schreibt (1859, pag. 596): „Der Spitze des Endospermkörpers ist der verhältnismäßig kleine, kugelige Embryo eingelagert. Er haftet durch einen fädlichen kurzen Träger, eine einfache Zellreihe, an der Innenwand des Embryosackes" und dazu entsprechende Figuren gegeben werden (1859, Taf. XVI, Fig. 4, 5), so liegt meines Erachtens kein Grund vor, solchen bestimmten Angaben nicht volle Beweiskraft zuzumessen.

Sicherlich sind weitere Untersuchungen wünschenswert. Die bisherigen Resultate lassen aber meines Erachtens voraussehen, daß weder die erneute und ergänzende Untersuchung von Helosis noch einer anderen Balanophoracee $\mathrm{zu}$ anderen Ergebnissen führen wird als zur Feststellung der Bildung eines an der Außenwand des Embryosackes haftenden Embryos, der aus der Eizelle hervorgeht, gelegentlich vielleicht auch aus einer anderen Zelle des Eiapparates - parthenogenetisch oder nach vorausgegangener Befruchtung.

Seit 1898 hat Balanophora als Beispiel eines besonders interessanten Typus der Apogamie bei Angiospermen gegolten. Trotz der außerordentlichen Zahl embryologischer Untersuchungen, die seither an Angiospermen der verschiedensten Verwandtschaftskreise ausgeführt worden sind und zur Aufdeckung größerer und kleinerer Variationen 
im Verlauf von Embryosack-, Embryo- und Endospermentwicklung geführt haben, hat sich dem von Treub und Lotsy beschriebenen Beispiel kein weiteres zugesellen wollen. Nunmehr ist erwiesen, daß dieses eine Beispiel selbst auf irrtümlicher Auffassung beruht, an Stelle der behaupteten Apogamie bei B. elongata und globosa Embryobildung aus der Eizelle und zwar aller Wahrscheinlichkeit nach somatische Parthenogenesis vorliegt. Man wird dieses Ergebnis vielleicht hier und dort im Interesse unserer phylogenetischen Anschauungen bedauern. Auch dem Verfasser dieser Mitteilung wäre der Nachweis weiterer Beispiele von Apogamie bei Angiospermen als Ergebnis mühevoller Untersuchungen willkommener gewesen.

Bei der jetzigen Sachlage wird man sich wohl mit dem Gedanken abfinden müssen, daß Embryobildung aus Zellen des Endosperms bei den Angiospermen nicht vorkommt. Bedenkt man aber, daß auch bei den Gymnospermen apogame Embryobildung völlig $\mathrm{zu}$ fehlen scheint, obschon deren primäres Endosperm dem apogame Embryonen liefernden Prothallium der Pteridophyten noch sehr viel näher steht als das sekundäre Endosperm der Angiospermen, so wird man diesen Verlust für die Phylogenie der Angiospermen doch nicht allzu schwer einzuschätzen haben.

\section{Zusammenfassung.}

Nach Treub (1898) und L otsy (1899) degenerieren im achtkernigen Embryosacke von Balanophora elongata und globosa nicht nur alle vier Kerne des Antipodenendes, sondern auch die drei Zellen des Eiapparates. Die ganze weitere Entwicklung ist auf die Teilungstätigkeit des oberen Polkernes beschränkt. Er liefert zunächst einen wenigzelligen Endospermkörper und von einer Zelle des letzteren soll dann schließlich nach der Darstellung der beiden Autoren die Mutterzelle des Embryos abgeteilt werden.

Bei der embryologisch-zytologischen Untersuchung verschiedener saprophytischer Angiospermen (Burmannia, Sciaphila, Cotylanthera) erhielt ich Präparate, welche ebenfalls apogame Embryobildung im Endosperm vermuten ließen, später aber durch den Nachweis somatischer Parthenogenesis eine viel einfachere Erklärung fanden. Dieses Ergebnis ließ auch für Balanophora eine von Treub und Lotsy übersehene Abstammung des Embryos von der Eizelle vermuten. Eine Nachuntersuchung hat dieser Vermutung Recht gegeben Zunächst hat meine Nachuntersuchung den von Treub und Lotsy für Balanophora elongata und globosa angegebenen Ent- 
wicklungsgang des Embryosackes bestätigt. Dieser entsteht entweder direkt aus der Embryosackmutterzelle oder nach einer einzigen Teilung derselben aus der oberen Tochterzelle, und zwar mit somatischer Chromosomenzahl der Kerne. Als richtig erwies sich ferner die von Treub und Lotsy in Übereinstimmung mit Van Tieghem gemachte Angabe, daß die Endospermbildung ausschließlich vom oberen Polkern ausgeht und der Embryosackraum nach einer ersten Teilung desselben in eine kleinere obere Endospermzelle und eine große Basaloder Haustorialzelle geteilt wird.

Die Regelmäßigkeit im nachfolgendèn Verlauf der Endospermbildung ist Treub und Lotsy entgangen. Durch drei aufeinanderfolgende Teilungsschritte entsteht aus der einen Endospermzelle zunächst ein achtzelliger, aus zwei vierzelligen Etagen bestehender Endospermkörper. Erst die nachfolgenden Teilungen finden mit wechselnder Richtung der Teilungswände statt und führen, namentlich in der Umgebung des Embryos, zur Bildung einer größeren Anzahl kleiner Zellen. Vom Eiapparat bleibt während der Endospermbildung die Eizelle erhalten. Vor der Weiterentwicklung zum Embryo nimmt sie zunächst an Größe ab und erfährt, wie die übrigen Elemente des Embryosackes, bei der Präparation Schrumpfungen. Treub und Lotsy haben hieraus den irrtümlichen Schluß auf eine völlige Degeneration des Eiapparates gezogen. In Wirklichkeit wächst aber die kleine Keimzelle während der Endospermbildung wieder stark heran. Der ersten Teilung ihres Kernes folgt eine Querteilung und nach wenigen weiteren Teilungen schlieft die Entwicklung des klein und undifferenziert bleibenden Embryos ab.

Aus dem Nachweis somatischer Parthenogenesis bei Balanophora elongata und globosa ergibt sich, daß die Angaben von Van Tieghem über das Vorkommen eines normalen Eiapparates und der Embryobildung aus einer befruchteten Eizelle bei Balanophora indica und ebenso die Angaben Hofmeister's über Bestäubung, Pollenschlauch- und Embryobildung bei Balanophora polyandra, fungosa und dioica mit Unrecht angezweifelt worden sind. Aus allen bisherigen Befunden an Balanophora-Arten sowie denjenigen Hofmeister's an Langsdorffia hypogaea, Sarcophyte sanguinea, Phyllocoryne jamaicensis usw. geht vielmehr hervor, daß der Embryo der Balanophoraceen seinen Ursprung meist ausder Eizelle, nur ausnahmsweise vielleicht auch aus einer anderen Zelle des Eiapparates nimmt, bei den meisten Vertretern der Familie nach vorausgegangener Befruch- 
tung, bei Balanophora elongata und globosa, bei Rhopalocnemis phalloides und Helosis guyanensis dagegen parthen ogenetisch.

\section{Literaturverzeichnis.}

Bernard, Ch., Recherches sur les sphères attractives chez Lilium candidum, Helosis guyanensis etc. Journal de Botanique 1900, T. XIV, pag. 206-212.

Ders., Sur l'embryogénie de quelques plantes parasites. Journal de Botanique 1903, T. XVII, pag. 23.

Brown, R., Description of the femal flower and fruit of Rafflesia Arnoldi, with remarks on its affinities; and an illustration of the structure of Hydnora africana, Juni 1834. Transact. Linn. Soc. of London 1842, Vol. XIX, pag. $221-247$.

Chodat, R. et Bernard,Ch., Sur le sac embryonnaire d'Helosis guyanensis. Journal de Botanique 1900, T. XIV, pag. 72-79.

Coulter, J. M. and Chamberlain, Ch. J., Morphology of Angiosperms. NewYork 1903.

Engler, A., Balanophoraceae, in Engler-Prantl, Natürl. Pflanzenfamilien 1889, III, 1, pag. 243-263 und Nachtr. III. pag. 99.

Engler, A. und Gilg, E., Syllabus der Pflanzenfamilien. VII. Aufl., Berlin 1912.

Ernst, A. und Bernard, Ch., Entwicklungsgeschichte des Embryosackes und des Embryos von Burmannia candida Engl. und B. Championii Thw. Ann. Jardin bot. Buitenzorg 1912, 2. Ser., Bd. X, pag. 161-188.

Dies., Entwicklungsgeschichte des Embryosackes, des Embryos und des Endosperms von Burmannia coelestis Don. Ann. Jardin bot. Buitenzorg 1912, 2. Ser., Bd. XI, pag. 234-257.

Goebel, K.. Organographie der Pflanzen. Jena 1898-1901.

Griffith, W., On the indian species of Balanophora, and on a new Genus of the family Balanophoreae. Transact. Linn. Society of London 1846, Vol. XX, pag. $93-108$.

Hofmeister, W., Neuere Beobachtungen über Embryobildung der Phanerogamen. Jahrb. f. wiss. Botanik I, 1858, pag. 110 und Taf. X. Fig. 6-13.

Ders., Neue Beiträge zur Kenntnis der Embryobildung der Phanerogamen. Abhandl. d. Sächs. Gesellschaft d. Wiss. 1859, Bd. VI, pag. 585, Taf. XIV u. XV.

J u el, H. O., Vergleichende Untersuchungen über typische und parthenogenetische Fortpflanzung bei der Gattung Antennaria. Kongl. Svenska VetenskapsAkademiens Handlingar 1900, Bd. XXXIII, No. 5.

Knuth, P., Handbuch der Blütenbiologie, bearbeitet von E. Loew und O. Appel 1904, Bd. III, 1. Teil; Balanophoraceae pag. 260-265.

Koorders, S. H., Exkursionsflora von Java. Dikotyledonen (Archichlamydeae) 1912, Bd. II.

Lotsy, J. P., Balanophora globosa Jungh., eine wenigstens örtlich verwitwete Pflanze. Ann. Jardin bot. Buitenzorg. 1899, Vol. XVI, pag. 174-186.

Ders., Rhopalocnemis phalloides Jungh., a morphological-systematical study. Ann. Jardin bot. Buitenzorg 1901, Vol. XVII, pag. 73-101. 
Ders., Vorträge über botanische Stammesgeschichte, III, 1. Jena 1911.

Miquel, F. A. G., Flora van Nederlandsch Indie, II, 1856.

Porsch, O., Versuch einer phylogenetischen Erklärung des Embryosackes und der doppelten Befruchtung der Angiospermen. Jena 1907.

Van Tieghem, Ph., Sur l'organisation florale des Balanophoracées etc. Bulletin Société botanique de France 1896, T. XIIII, pag. 295-310.

Ders., Sur les Inovulées. Ann. sc. nat. Bot. 1907, 9. Sér., T. VI, pag. 125-260.

Tischler, G., Über die Entwicklung der Samenanlagen in parthenokarpen Angiospermenfrüchten. Jahrb. f. wiss. Bot. 1913, Bd. LII, pag. 1-84.

Treub, M., L'organe femelle et l'apogamie du Balanophora elongata Bl. Ann. Jardin bot. Buitenzorg 1898, T. XV, pag. 1-25.

Samuelss on, G., Studien über die Entwicklungsgeschichte der Blüten einiger Bicornestypen. Svensk Botanisk Tidskrift 1913, Bd. VII, pag. 97-188.

Strasburger, Ed., Einige Bemerkungen zur Frage nach der ,doppelten Befruchtung" bei den Angiospermen. Bot. Zeitg. 1900, Bd. LVIII, pag. 293-316.

von Wettstein, R., Handbuch der systematischen Botanik, II. Aufl., Wien 1911.

Winkler, H., Über Parthenogenesis und Apogamie im Pflanzenreiche. Progressus rei bot. 1908, Bd. II, pag. 293-454.

Ders., Apogamie und Parthenogenesis. Handwörterbuch der Naturwissenschaften, 1913, Bd. IV, pag. 265.

Wirz, H., Beiträge zur Entwicklungsgeschichte von Sciaphila spec. und von Epirrhizanthes elongata Bl., 1910, Bd. CI, pag. 395-446.

\section{Figurenerklärung zu Tafel I und II.}

\section{Tafel I.}

Alle Figuren dieser Tafel sind mit Zeiss Apochromat $2 \mathrm{~mm}$ n. Ap. 1.30, Tubus $160 \mathrm{~mm}$ und dem Leitzschen Zeichenokular gezeichnet worden. Vergr. 620:1.

Fig. 1-12: B a lan ophora gl obosa Jungh.

Fig. 1. Embryosackmutterzelle mit vakuoligem, infolge der Präparation leicht kontrahiertem Plasma. Kern in Vorbereitung zur ersten Teilung.

Fig. 2. Kernteilung (Dispiremstadium) am Basalende der Embryosackmutterzelle.

Fig. 3. Zweikernige Embryosackzelle mit Vakuolenbildung im Plasma zu Seiten der Kerne.

Fig. 4. Stark gewachsener, zweikerniger Embryosack nach Ausbildung der zentralen Vakuole. Plasma am unteren Pole besonders dicht, erste Andeutung der Bildung des emporwachsenden Embryosackzweiges.

Fig. 5. Tochterzellen, durch äquale Teilung aus der Embryosackmutterzelle hervorgegangen. Plasmahäute deutlich voneinander entfernt, eine Zellulosewand zwischen den beiden Zellen indessen nicht nachweisbar. Beide Zellen mit gleichartig differenziertem Inhalt.

Fig. 6. Embryosackmutterzelle kurz nach vollzogener Teilung in eine kleinere untere und eine größere, obere Zelle. 
Fig. 7. Entwicklung der oberen der beiden Tochterzellen zum Embryosacke.

Fig. 8. Zweikerniger Embryosack mit ungefähr gleich starker Ausbildung der beiden aufwärts gerichteten Äste.

Fig. 9. Vierkerniger Embryosack. Die Kerne paarweise an den Enden der beiden Äste gelagert. Im Plasma des zukünftigen Antipodenendes eine Vakuole.

Fig. 10. Achtkerniger Embryosack kurz nach der Ausbildung des Eiapparates. Der den Eiapparat erzeugende Ast ist über den ursprünglichen Scheitel des Embryosackes hinausgewachsen. Die beiden Synergiden enthalten in dichtem, vakuolenfreiem Plasma scheitelwärts die kleinen Kerne. Eikern und oberer Polkern von bedeutenderer Größe und mit deutlichem Kernkörperchen. Am Antipodenende ist die Zellbildung unterblieben. Alle vier Kerne sind gleich groß, von übereinstimmender Struktur und liegen, vom Scheitel des Astes in der Regel durch eine Vakuole getrennt, in einem breiten Plasmabande.

Fig. 11 u. $11 a$. Achtkerniger Embryosack nach völliger Ausbildung des Eiapparates. Die vier Kerne des Antipodenendes dicht aneinander geschmiegt in einem Plasmagürtel. Am anderen Pole (Fig. 11) die beiden Synergiden ( $s$ ) und der große obere Polkern $(o P)$. In Fig. $11 a$ die Eizelle (e), die sich von den Synergiden durch bedeutendere Größe und einen ebenfalls größeren, ein Kernkörperchen aufweisenden Kern auszeichnet.

Fig. 12. Oberer Teil eines Embryosackes mit den vier freien Kernen im Antipodenaste $(a k)$. Eizelle $(e)$ mit oberem Polkern $(o P)$ im anderen Aste. Die unter der Eizelle gelegenen Synergiden sind in der Figur nicht eingezeichnet.

Fig. 13-18: Balanophora elongata Bl.

Fig. 13. Embryosackscheitel mit gut entwickelter Eizelle $(e)$ und den beiden degenerierenden Synergiden. Unter der Eizelle reichliches, vakuoliges Embryosackplasma mit dem oberen Polkern $(o P)$.

Fig. 14. Embryosackscheitel vor der ersten Teilung des oberen Polkerns. Eizelle (e) mit stark geschrumpftem Plasma aber deutlichem Kern mit Nukleolus. Polkern stark gewachsen, mit drei Nukleolen (in der Zeichnung davon nur zwei eingetragen), stark gefärbten Chromatinkörnern, in vakuoligem Sackplasma eingebettet.

Fig. 15. Eizelle $(e)$ und Reste der beiden Synergiden. In der einen der Kern durch Fragmentation in drei Stücke zerfallen. Unter dem Eiapparat dichtes Plasma. Oberer Polkern in T'eilung, ca. 16 Chromosomen.

Fig. 16. Eiapparat mit degenerierenden Synergiden und erhalten bleibender Eizelle $(e)$. Darunter die erste Endospermzelle $(E)$ mit großem Kern und vakuolenreichem Plasma. Der kontrahierte Wandbelag der großen Basalzelle mit ihrem Kern ist in der Figur nicht eingezeichnet.

Fig. 17. Scheitel des Embryosackes nach der zweiten Endospermzellteilung. Die erste Endospermzelle ist durch eine Längsteilung in zwei nebeneinander liegende Zellen $(E)$ geteilt worden. Darunter der dünne Wandbelag der Basalzelle mit großem Kern $(B k)$. Am Scheitel des Embryosackes Reste der beiden Synergiden und die Eizelle (e).

Fig. 18. Scheitel eines Embryosackes mit vier Endospermzellen, die durch den dritten Teilungsschritt entstanden sind. Darunter Kern und Stück des Wandbelages der Basalzelle. Am Scheitel selbst die Reste der Synergiden und die Eizelle (e), die zwischen die oberen Endospermzellen eingesenkt ist. 
Flora Band 106.

Taf.I.

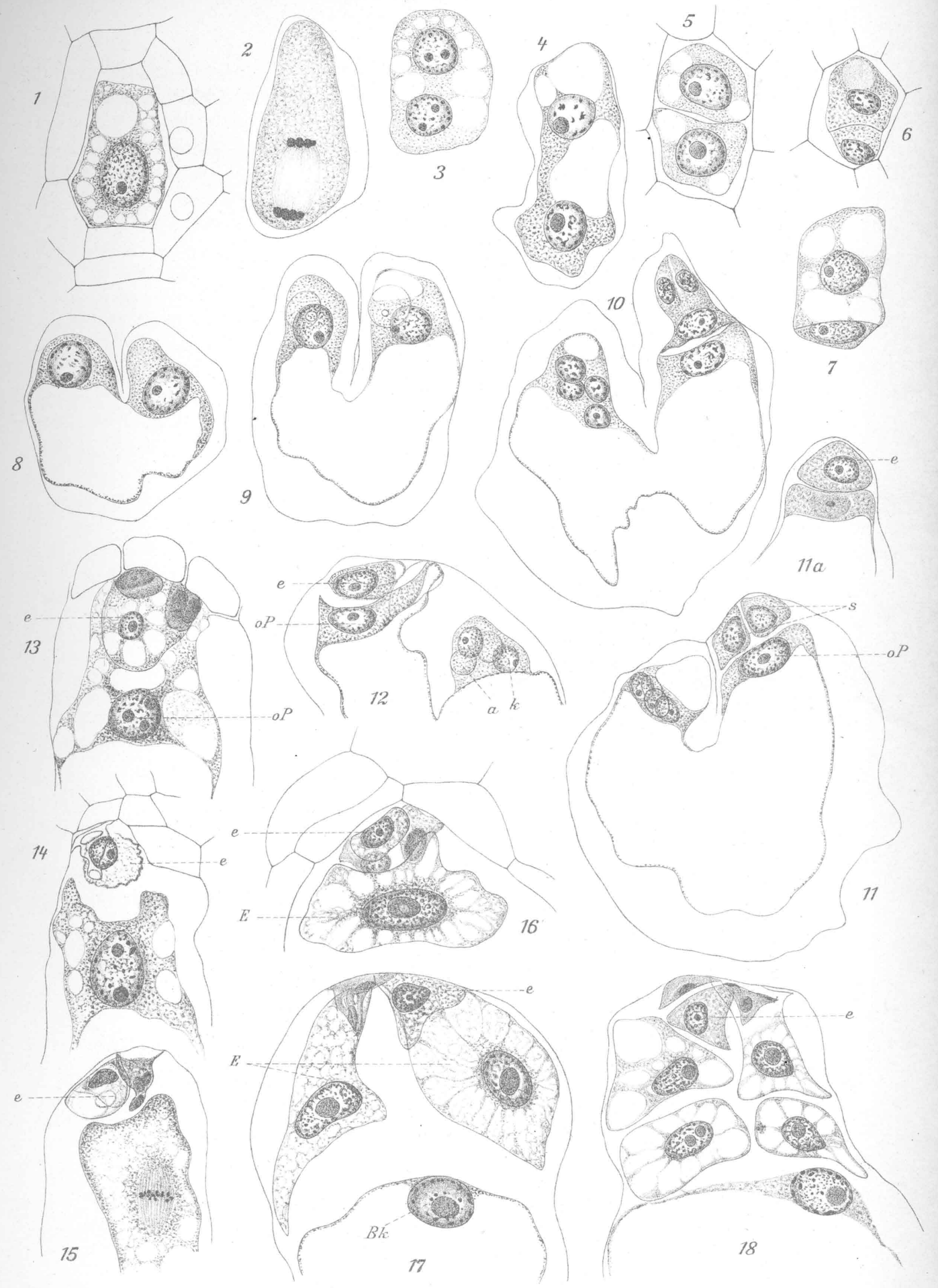

A. Errust gez.

Verlag von Gustav Fischer in Jena. 
Flora Band 106.

Taf. II.

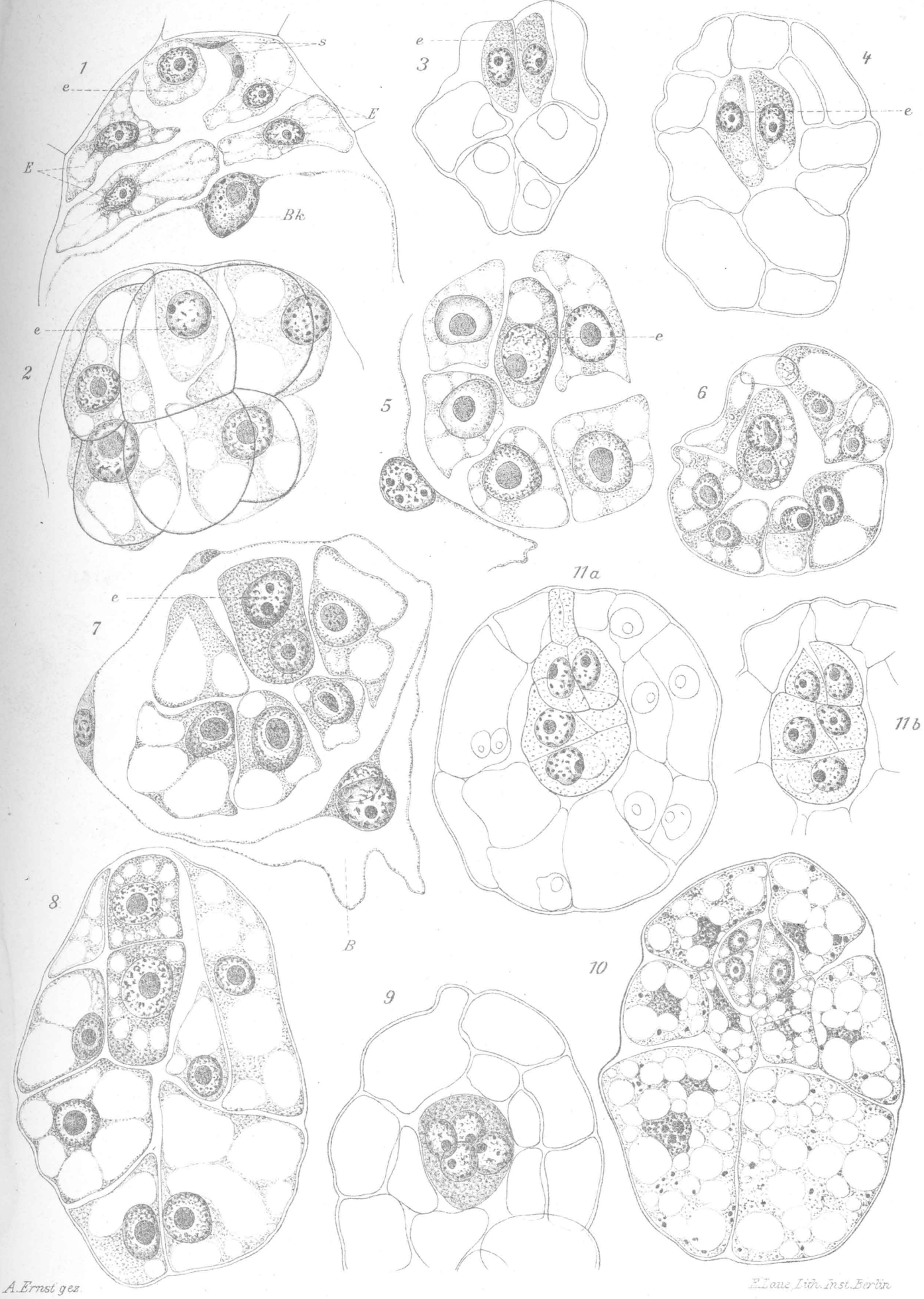

Verlag von Gustav Fischer in Jena 


\section{Taf. II.}

Die Figuren 1, 2, 5, 7-9 sind mit Zeiss Apochromat $2 \mathrm{~mm}$ n. Ap. 1.30, Tubus $160 \mathrm{~mm}$ und dem Zeichenokular von Leitz gezeichnet worden. Vergr. 620:1. Die Figuren 3, 4, 6, 10 und 11 sind mit Leitz Objektiv 7 und dem Zeichenokular von Leitz gezeichnet worden. Vergr. 420:1.

Figur 1-4: Balanophora elongata Bl.

Fig. 1. Embryosackscheitel mit Embryo- und Endospermbildung. Eizelle (e) mit vakuoligem Plasma und großem Kern, Reste der $(s)$ Synergiden zwischen den Zellen der oberen Etage des Endosperms eingesenkt. Von den je vier Zellen der beiden Endosperm-Zelletagen sind nur die zwei bei oberer Einstellung sichtbaren Zellen $(E)$ gezeichnet worden. Unter der Endospermzellgruppe ein Stück des Wandbelages der großen Basalzelle mit Kern $(B k)$.

Fig. 2. Acht Endospermzellen in zwei vierzelligen Etagen. Zwischen den Zellen der oberen Etage ist bei mittlerer Einstellung die langgestreckte Keimzelle (e) wahrnehmbar.

Fig. 3 und 4. Vorgeschrittenere Stadien der Endospermbildung mit jungen Embryonen (e). Von den Endospermzellen sind nur die bei mittlerer Einstellung, gleichzeitig mit dem Embryo sichtbaren, gezeichnet worden. In Fig. 3 finden sich unmittelbar am Scheitel, in Fig. 4 unter einer obersten Endospermzelle zwei nebeneinander liegende Zellen, die durch Färbung von Plasma und Kern von den Endospermzellen verschieden sind. Sie sind entweder durch Längsteilung aus der einen Keimzelle oder durch Weiterentwicklung von zwei Zellen des Eiapparates zu Keimzellen entstanden.

Fig. 5-11: Balanophora globosa Jungh.

Fig. 5. Endospermkörper im Medianschnitt mit Keimzelle (e) und Wandbelag der Basalzelle. Die bis an den Scheitel des Sackes reichende Keimzelle unterscheidet sich in Struktur von Kern und Plasma von den Endospermzellen.

Fig. 6. Keimzelle zweikernig, rings von einer Schicht von Endospermzellen umschlossen.

Fig. 7. Medianschnitt durch einen Embryosack. Zweikernige, scheitelwärts freie Keimzelle (e) mit seitlich und basalwärts sich anschließenden Endospermzellen. Basalzelle durch das Wachstum des Endosperms schon stark zusammengedrängt, mit zwei Kernen in dem an das Endosperm anschließenden Teil des Wandbelages.

Fig. 8. Durch eine Querwand zweigeteilter Embryo, scheitelwärts bis an die Wand des Embryosackes reichend. Seitlich und gegen die Basis des Embryosackes schließen sich große Endospermzellen mit vakuoligem Plasma an.

Fig. 9. Vierkerniger Embryo inmitten des Endospermkörpers.

Fig. 10. Endosperm und Embryo aus einem fast ausgereiften Samen. Embryo ohne Suspensor, fast kugelig, von einer Endospermschicht umhüllt, durch zwei Längswände in Quadranten geteilt. Von diesen (in der Zeichnung nur zwei gezeichnet) der eine durch eine Querwand wieder in zwei Zellen geteilt. Endospermzellen mit stark vakuoligem Plasma. In diesem Eiweißkörner und der degenerierende Zellkern.

Fig. $11 a$ und $b$. Endosperm mit Embryo aus einem fast reifen Samen. Embryo aus drei zweizelligen Etagen und einer schmalen, inhaltsarmen und schon kernlos gewordenen Suspensorzelle bestehend. Die letztere reicht zwischen den Endospermzellen hindurch bis an die Oberfläche des Embryosackes. Fig. $11 a$ bei tiefer, Fig. $11 b$ bei hoher Einstellung gezeichnet. 